\title{
Lower limb lymphedema in lung adenocarcinoma: Two case reports
}

\author{
MISAE SHINODA ${ }^{1}$, HARUKA AKUTSU ${ }^{1}$, TOSHIHIRO OHTANI ${ }^{2}$, TOMOHIRO TAMURA $^{3}$ and HIROAKI SATOH ${ }^{3}$ \\ Divisions of ${ }^{1}$ Nursing, ${ }^{2}$ Pharmacy and ${ }^{3}$ Respiratory Medicine, Mito Medical Center, \\ University of Tsukuba, Mito, Ibaraki 310-0015, Japan
}

Received May 17, 2016; Accepted July 4, 2016

DOI: $10.3892 / \operatorname{mco} .2016 .988$

\begin{abstract}
Breast and gynecological cancers and their treatment may cause lymphedema of the upper and lower extremities, respectively. We herein report the cases of two patients with lung adenocarcinoma who developed lymphedema of the lower extremities. One patient harbored an epidermal growth factor receptor mutation and the other patient harbored an anaplastic lymphoma kinase fusion gene. The patients had developed intra-abdominal lymph node metastases and received several lines of chemotherapy. In both patients, lymphedema in the lower extremities developed $>30$ months after the initiation of first-line chemotherapy. To the best of our knowledge, these are the first reported cases of lung cancer patients who developed lymphedema in the lower extremities. Although rarely, lymphedema of the lower extremities may develop long after successful therapy with molecular-targeted therapy in advanced lung adenocarcinoma patients with a specific genetic etiological background.
\end{abstract}

\section{Introduction}

Cancer-related secondary lymphedema is a pathological condition that develops following obstruction of the lymphatic system by direct invasion, metastasis, surgical removal, or irradiation of the lymph nodes (1). Lymphedema may occur months to years following treatment for breast and gynecological cancers with lymph node removal, or obstruction of the lymphatic system by irradiation or metastases $(1,2)$. We recently encountered two advanced lung adenocarcinoma patients with lower limb lymphedema, who achieved long-term survival with epidermal growth factor receptor (EGFR)-tyrosine kinase inhibitors (TKIs) or anaplastic lymphoma kinase (ALK)-TKIs. To the best of our knowledge, these are the first advanced lung adenocarcinoma cases who developed lymphedema in the lower extremities long after receiving molecular-targeted therapies.

Correspondence to: Professor Hiroaki Satoh, Division of Respiratory Medicine, Mito Medical Center, University of Tsukuba, 2-3-7 Miya-Machi, Mito, Ibaraki 310-0015, Japan

E-mail: hirosato@md.tsukuba.ac.jp

Key words: lower limb lymphedema, lung adenocarcinoma

\section{Case reports}

Case 1. A 63-year-old female patient was diagnosed with T2aN3M1b lung adenocarcinoma with an EGFR mutation (exon 19 deletion). The patient had received 5 types of chemotherapeutic regimens, including two types of EGFR-TKIs. At 33 months after therapy initiation, the patient developed intra-abdominal lymph node, pelvic bone and left femoral metastases, and severe edema in the left leg (Fig. 1). Within a 2-week period, the edema expanded to involve the right leg. The patient was diagnosed with lymphedema due to lymphatics obstruction by the intra-abdominal lymph node, pelvic bone and femoral metastases. No edema was observed in other sites, including the trunk, upper extremities and face. Radiation therapy for the metastatic sites was unsuccessful in controlling lymphedema and the patient succumbed to lung adenocarcinoma 2 months after the development of lymphedema.

Case 2. A 41-year-old male patient was diagnosed with T4N3M1b lung adenocarcinoma with an ALK fusion gene. The patient had received 4 types of chemotherapeutic regimens, including two types of ALK inhibitors. At 37 months after the initiation of therapy, the patient developed liver and intra-abdominal lymph node metastases, with edema in both lower limbs. The patient was diagnosed with lymphedema due to lymphatics obstruction by intra-abdominal lymph node metastases. There was no edema in other sites, including the trunk, upper extremities and face. No specific therapy was successful in controlling lymphedema and the patient succumbed to lung adenocarcinoma 1 month after the development of lymphedema.

\section{Discussion}

Survival among patients with advanced lung adenocarcinoma has steadily improved (3). The cornerstone of management for lung adenocarcinoma with a specific genetic etiology, such as EGFR mutations and ALK fusion gene, is molecular-targeted therapy, with the aim being long-term administration. Therefore, survival, particularly in those with a specific genetic etiology, has significantly improved (3-5). Improvements in survival, however, may be accompanied by long-term sequelae, including meningitis carcinomatosa, an issue that has recently attracted attention $(6,7)$.

Cancer-related secondary lymphedema of the extremities is a pathological condition that develops following obstruction 


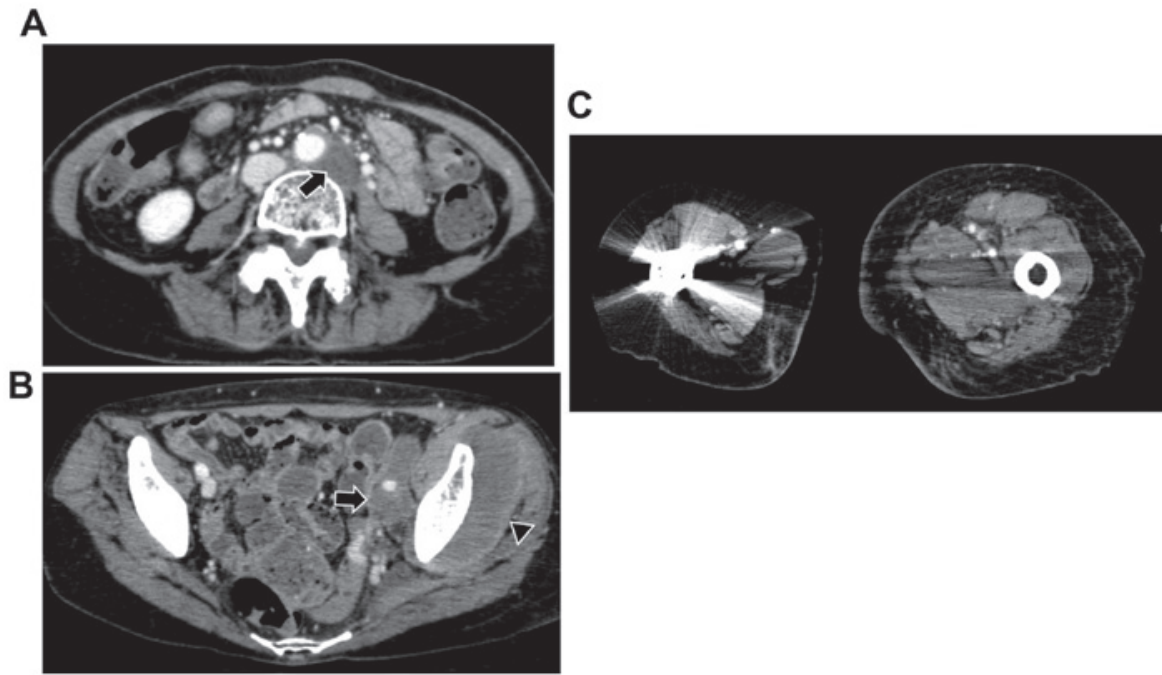

Figure 1. Computed tomography scan in Case 1 showing (A and B) intra-abdominal lymph node metastases, (arrows); (B) metastasis to the pelvis, (arrowhead); and $(\mathrm{C})$ severe edema in both legs.

of the lymphatic system by direct invasion or metastasis, or treatments such as surgical removal or irradiation (1). However, lymphedema, which is acute or chronic edema associated with disruption of the lymphatic system, is a perplexing and poorly understood clinical entity $(1,2)$. It is well-documented in the literature that patients with breast cancer experience lymphedema of the upper extremities secondary to surgical resection of lymph vessels and nodes, from radiation or obstruction of the lymphatic system by metastatic cancer (1). Petrek and Heedan reported that the incidence of lymphedema in breast cancer patients ranged from 6 to $30 \%$ (8). The incidence may vary according to the source of patients, duration of follow-up and measurement techniques; in addition, the definition of lymphedema may vary across studies. In general, reports with shorter follow-up reported lower incidences of lymphedema (8). Patients with gynecological cancers experience lymphedema in the lower extremities $(1,2)$. Beesley et al reported that approximately one-fifth of ovarian cancer patients develop lymphedema (9). Rehabilitation therapy for post-treatment effects from cancer and its treatment is required to improve functioning and quality of life (10). In patients with distant metastatic recurrence and a poor performance status, however, rehabilitation may be challenging.

We herein report two cases of patients with advanced lung adenocarcinoma who developed lymphedema in the lower extremities following long-term survival with the use of EGFR-TKIs or ALK-TKIs. Both patients had developed intra-abdominal lymph node metastases, which may have caused obstruction of the lymphatic system. No therapy was able to control lymphedema and the patients succumbed to the disease shortly after the development of lymphedema. Metastases to lower parts of the body, including the intra-abdominal lymph nodes, may increase the risk of lymphedema by further lymphatics obstruction in these patients. To the best of our knowledge, these are the first two reported cases of lymphedema in the lower extremities following molecular-targeted therapies for advanced non-small-cell lung cancer (NSCLC). It should be kept in mind that lymphedema in the lower extremities may develop long after successful therapy with molecular-targeted therapy in advanced NSCLC patients with a specific genetic etiology.

\section{References}

1. Jakes AD and Twelves C: Breast cancer-related lymphoedema and venepuncture: A review and evidence-based recommendations. Breast Cancer Res Treat 154: 455-461, 2015.

2. Casey C, Chen LM and Rabow MW: Symptom management in gynecologic malignancies. Expert Rev Anticancer Ther 11: 1077-1089, 2011.

3. Bareschino MA, Schettino C, Rossi A, Maione P, Sacco PC, Zeppa R and Gridelli C: Treatment of advanced non small cell lung cancer. J Thorac Dis 3: 122-133, 2011.

4. Thomas A, Liu SV, Subramaniam DS and Giaccone G: Refining the treatment of NSCLC according to histological and molecular subtypes. Nat Rev Clin Oncol 12: 511-526, 2015.

5. Gerber DE, Gandhi L and Costa DB: Management and future directions in non-small cell lung cancer with known activating mutations. Am Soc Clin Oncol Educ Book 2014: e353-e365, 2014.

6. Togashi Y, Hayashi H, Nakagawa K and Nishio K: Clinical utility of erlotinib for the treatment of non-small-cell lung cancer in Japanese patients: Current evidence. Drug Des Devel Ther 8: 1037-1046, 2014.

7. Le Rhun E, Taillibert S and Chamberlain MC: Carcinomatous meningitis: Leptomeningeal metastases in solid tumors. Surg Neurol Int 4 (Suppl 4): S265-S288, 2013.

8. Petrek JA and Heelan MC: Incidence of breast carcinoma-related lymphedema. Cancer 83 (12 Suppl American): S2776-S2781, 1998.

9. Beesley V, Janda M, Eakin E, Obermair A and Battistutta D: Lymphedema after gynecological cancer treatment: Prevalence, correlates and supportive care needs. Cancer 109: 2607-2614, 2007.

10. Loh SY and Musa AN: Methods to improve rehabilitation of patients following breast cancer surgery: A review of systematic reviews. Breast Cancer (Dove Med Press) 7: 81-98, 2015. 